Research Paper

\title{
In Search for Pheromone Receptors: Certain Members of the Odorant Receptor Family in the Desert Locust Schistocerca gregaria (Orthoptera: Acrididae) Are Co-expressed with SNMP1
}

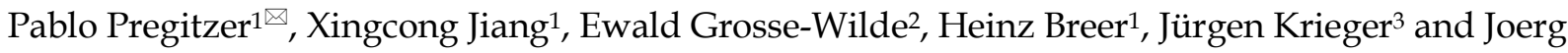 \\ Fleischer ${ }^{3 凶}$ \\ 1. University of Hohenheim, Institute of Physiology (230), Stuttgart, Germany \\ 2. Max Planck Institute for Chemical Ecology, Department of Evolutionary Neuroethology, Jena, Germany \\ 3. Martin Luther University Halle-Wittenberg, Institute of Biology/Zoology, Department of Animal Physiology, Halle (Saale), Germany \\ $\triangle$ Corresponding author: Pablo Pregitzer, University of Hohenheim, Institute of Physiology (230), Stuttgart, Germany, phone: +4971145922270 , fax: +4971145923726 , \\ email: p_pregitzer@uni-hohenheim.de and Joerg Fleischer, Martin Luther University Halle-Wittenberg, Institute of Biology/Zoology, Department of Animal Physiology, \\ Halle (Saale), Germany, phone: +49345 5526476, fax: +49345 5527152, email: joerg.fleischer@zoologie.uni-halle.de \\ (C) Ivyspring International Publisher. This is an open access article distributed under the terms of the Creative Commons Attribution (CC BY-NC) license
} (https://creativecommons.org/licenses/by-nc/4.0/). See http://ivyspring.com/terms for full terms and conditions.

Received: 2016.11.18; Accepted: 2017.03.09; Published: 2017.07.15

\begin{abstract}
Under given environmental conditions, the desert locust (Schistocera gregaria) forms destructive migratory swarms of billions of animals, leading to enormous crop losses in invaded regions. Swarm formation requires massive reproduction as well as aggregation of the animals. Pheromones that are detected via the olfactory system have been reported to control both reproductive and aggregation behavior. However, the molecular basis of pheromone detection in the antennae of Schistocerca gregaria is unknown. As an initial step to disclose pheromone receptors, we sequenced the antennal transcriptome of the desert locust. By subsequent bioinformatical approaches, 119 distinct nucleotide sequences encoding candidate odorant receptors (ORs) were identified. Phylogenetic analyses employing the identified ORs from Schistocerca gregaria (SgreORs) and OR sequences from the related species Locusta migratoria revealed a group of locust ORs positioned close to the root, i.e. at a basal site in a phylogenetic tree. Within this particular OR group (termed basal or b-OR group), the locust OR sequences were strictly orthologous, a trait reminiscent of pheromone receptors from lepidopteran species. In situ hybridization experiments with antennal tissue demonstrated expression of b-OR types from Schistocerca gregaria in olfactory sensory neurons (OSNs) of either sensilla trichodea or sensilla basiconica, both of which have been reported to respond to pheromonal substances. More importantly, two-color fluorescent in situ hybridization experiments showed that most b-OR types were expressed in cells co-expressing the "sensory neuron membrane protein 1" (SNMPI), a marker indicative of pheromone-sensitive OSNs in insects. Analyzing the expression of a larger number of SgreOR types outside the b-OR group revealed that only a few of them were co-expressed with SNMPI.

In summary, we have identified several candidate pheromone receptors from Schistocerca gregaria that could mediate responses to pheromones implicated in controlling reproduction and aggregation behavior.
\end{abstract}

Key words: olfaction, insect, pheromone receptor, desert locust, antenna, sensory neuron membrane protein 1

\section{Introduction}

By means of various cuticular hair-like structures (sensilla) on their antennae, insects are capable of detecting a variety of volatile chemicals in their surroundings, including pheromone compounds that are used to exchange important information between conspecifics and shape distinct behaviors such as aggregation, mate finding and courtship $(1,2)$. To date, our knowledge about the molecular mechanisms 
underlying the reception of pheromones is mainly based on studies of holometabolic insects, especially lepidopteran and dipteran species (3-6). In Lepidoptera and Diptera, pheromone detection appears to be mainly assigned to trichoid sensilla (6-8), which typically harbor the dendritic processes of up to three olfactory sensory neurons (OSNs) (9-13). In these pheromone-responsive sensilla, at least one OSN responds to a pheromonal compound and comprises the molecular machinery for pheromone signaling, including a specific odorant receptor (OR) $(6,14,15)$, the odorant receptor co-receptor (Orco) (16-19) as well as the "sensory neuron membrane protein 1" (SNMP1) (20-25).

In marked contrast to lepidopteran and dipteran species, the mechanisms of pheromone signaling in hemimetabolic insects are largely elusive although some pheromonal compounds have been identified in locusts (26-32). The hemimetabolic locusts are only distantly related to holometabolic insects (33). Moreover, some locust species (in particular the desert locust Schistocerca gregaria) are unique for a striking phase polyphenism including a change between a solitary and a gregarious phase $(1,34)$. In Schistocerca gregaria, animals in the gregarious phase can form huge swarms that tremendously threaten agricultural crops in Africa and Asia. Swarm formation is based on massive reproduction as well as aggregation. In locusts, both reproductive and aggregation behaviors are supposed to involve pheromones $(1,27,32,35,36)$ and evidence has been accumulated that such pheromones elicit responses in antennal sensilla (31,37-39). The antennal sensilla of locusts are categorized in distinct classes with the slender sensilla trichodea containing one to three OSNs and the broader and more massive sensilla basiconica comprising between 20 to 50 sensory neurons. As a third type, sensilla coeloconica (pegs in pits) contain one to four OSNs (40). In Schistocerca gregaria, OSNs in sensilla coeloconica do not express Orco or SNMP1 $(41,42)$ but comprise variant ionotropic receptors (43) that mediate responses to distinct classes of odorants (carboxylic acids, amines, ammonia) in other insects $(44,45)$. In contrast, based on the expression of Orco, OSNs in sensilla trichodea and sensilla basiconica of the desert locust most likely express ORs for odorant detection. Moreover, some OSNs in these types of sensilla were also found to express SNMP1 $(41,42)$. Since SNMP1 is considered indicative of pheromone-responsive neurons in other insects $(20-22,25,46,47)$, SNMP1-expressing OSNs in Schistocerca gregaria might also respond to pheromones and supposedly express appropriate pheromone-binding OR types. In view of the indicated roles of pheromones in locust aggregation and reproductive behaviors and with regard to the potential of using a blockade of pheromone signaling for insect control strategies, a better understanding of pheromone reception in the swarm-forming crop pest Schistocerca gregaria is highly desirable. However, until now, receptors for pheromones in locusts are still elusive and in view of the sparse information about candidate pheromone compounds, experimental approaches to identify pheromone receptors are limited. Therefore, as an initial step to identify OR types in Schistocerca gregaria (SgreORs) activated by pheromonal compounds, the present study was based on the paradigm that candidate pheromone receptors should be expressed in SNMP1-positive neurons of locust antennae. Towards this goal, we have screened a Schistocerca gregaria antennal transcriptome database for candidate OR-encoding sequences and subsequently determined their topographic expression pattern in the antennae with a particular emphasis on a possible co-expression with SNMP1.

\section{Materials and Methods}

\section{Animals and tissue treatment}

Adult Schistocerca gregaria were purchased from Bugs International (Irsingen/Unterfeld, Germany) and their antennae were dissected using autoclaved surgical scissors. For RNA extraction, antennae were immediately frozen in liquid nitrogen and stored at $-70{ }^{\circ} \mathrm{C}$. For in situ hybridization experiments, antennae were embedded in Tissue-Tek O.C.T. Compound (Sakura Finetek, Alphen aan den Rijn, The Netherlands).

\section{Identification of OR-encoding sequences from Schistocerca gregaria by transcriptome sequencing and bioinformatical analyses}

From the antennae of adult male and female desert locusts, total RNA was extracted using Trizol reagent (Thermo Fisher Scientific, Waltham, MA, USA) following the manufacturer's protocol. The material was sent to the Max Planck-Genome-centre (Cologne, Germany) where a TruSeq RNA library was generated. The library was sequenced on a HiSeq2500 (Illumina, San Diego, CA, USA), generating a total of $51,151,235$ paired end 100 base pair (bp) reads. The data were cleaned and trimmed by the Max Planck-Genome-centre. The results were assembled in CLC Genomics Workbench 8 (Qiagen, Venlo, The Netherlands) using the de novo assembler algorithm with default options (yet, all contigs below $300 \mathrm{bp}$ size were omitted). This resulted in 55,060 contigs with an N50 of 2,223 bp. To identify candidate OR sequences, contigs were analyzed with blastx searches using databases of known OR-coding sequences in 
Geneious 7 (Biomatters, Auckland, New Zealand). Transcripts with E-values below $10^{-3}$ were extracted and assembled with the Geneious assembler under highest similarity settings to reduce redundancy. The resultant contigs and unique sequences were manually annotated using standard blastx comparisons with the nr database (NCBI, Bethesda, MD, USA). Using both tBLASTx and BLASTp methods, the latter sequences were subsequently utilized as secondary queries to identify additional OR-encoding sequences present in the transcriptome database. Next, verification of the identified sequences as putative OR-encoding sequences was accomplished via BLASTx survey based on a NCBI non-redundant protein database.

\section{Reverse transcription polymerase chain reaction (RT-PCR)}

Total RNA was extracted from antennae of adult males and females using Trizol reagent (Thermo Fisher Scientific) following the manufacturer's protocol. Poly(A)+ RNA was isolated from $100 \mu \mathrm{g}$ total RNA with oligo(dT)25 magnetic beads (Thermo Fisher Scientific) according to the supplier's specifications and with a final elution in $30 \mu \mathrm{l} \mathrm{H}_{2} 0$. Poly $(\mathrm{A})^{+}$RNA was converted into cDNA utilizing 10 $\mu \mathrm{l}$ poly(A) ${ }^{+}$RNA elution, $4 \mu \mathrm{l}$ first strand buffer (250 $\mathrm{mM}$ Tris pH 8.3, $375 \mathrm{mM} \mathrm{KCl}, 15 \mathrm{mM} \mathrm{MgCl} 2), 1 \mu l 10$ $\mathrm{mM}$ dNTP mix, $1 \mu \mathrm{l}$ RNaseOUT recombinant ribonuclease inhibitor, $2 \mu$ 1,4-dithiothreitol (DTT) (0.1M), $1 \mu$ oligo(dT) 18 primer and $1 \mu$ Superscript III reverse transcriptase (Thermo Fisher Scientific). Synthesis of cDNA was conducted at $55^{\circ} \mathrm{C}$ for $50 \mathrm{~min}$ followed by incubation at $70{ }^{\circ} \mathrm{C}$ for $15 \mathrm{~min}$. Non-quantitative RT-PCR was performed using SgreOR-specific sense and antisense primers (supplementary table 1). For PCR amplification of SgreOR-encoding sequences, the following conditions were used: $94^{\circ} \mathrm{C}$ for $90 \mathrm{~s}$ followed by 20 cycles with 94 ${ }^{\circ} \mathrm{C}$ for $30 \mathrm{~s}, 50-60{ }^{\circ} \mathrm{C}$ for $30 \mathrm{~s}$ (thereby, the annealing temperature was decreased by $0.5^{\circ} \mathrm{C}$ per cycle) and 72 ${ }^{\circ} \mathrm{C}$ for $90 \mathrm{~s}$. Subsequently, 20 cycles with an annealing temperature of $40-50{ }^{\circ} \mathrm{C}$ were performed followed by incubation at $72{ }^{\circ} \mathrm{C}$ for $15 \mathrm{~min}$. Alternatively, PCR reactions were run at $97^{\circ} \mathrm{C}$ for $1 \mathrm{~min}$ followed by 34 cycles with $97^{\circ} \mathrm{C}$ for $40 \mathrm{~s}$ and $68^{\circ} \mathrm{C}$ for $3 \mathrm{~min}$. After the last cycle, a final incubation at $68{ }^{\circ} \mathrm{C}$ for 3 min was performed. PCR products were run on $1 \%$ agarose gels and visualized by staining with ethidium bromide. PCR products of the expected size were subsequently purified using the geneclean kit (MP Biomedicals, Santa Ana, CA, USA) and cloned into the pGEM-T or the pGEM-T easy vector (Promega, Madison, WI, USA). Vectors carrying putative OR sequences were sequenced by Macrogen (Amsterdam,
The Netherlands) and analyzed utilizing the Chromas Software (http:/ / technelysium.com.au/wp/chromas; Technelysium, South Brisbane, Australia).

\section{Phylogenetic analyses}

An amino acid alignment comprising the identified OR sequences from Schistocerca gregaria (SgreOR; this study) and the recently described OR sequences from Locusta migratoria (LmigOr) (48) was created using BioEdit (http://www.mbio.ncsu.edu/ BioEdit/bioedit.html) and MEGA 6.06 software (49). Thereby, two SgreORs and four LmigOrs that represented only very short sequences were not included. Next, a neighbor-joining tree was constructed based on a Clustal $\mathrm{X}$ alignment utilizing the MEGA 6.06 software and the default settings of this software package. Finally, the tree was rooted using sequences from several insect species (Schistocerca gregaria, Locusta migratoria, Manduca sexta, Drosophila melanogaster and Anopheles gambiae) encoding the odorant receptor co-receptor Orco, an unusual member of the OR family that is highly conserved across insect orders $(16,17)$.

\section{In situ hybridization}

Digoxigenin-labeled or biotin-labeled antisense probes were synthesized from linearized pGEM-T vectors containing partial or full length coding sequences of SgreORs using the T7/Sp6 RNA transcription system (Roche Diagnostics, Mannheim, Germany) following the manufacturer's protocol. Antennae of male and female adult Schistocerca gregaria locusts were crosscut into two halves, embedded in Tissue-Tek O.C.T. Compound and used to make $12 \mu \mathrm{m}$ thick longitudinal sections with a Jung CM300 or a Leica CM3050 S cryostat (Leica Microsystems, Bensheim, Germany) at $-21{ }^{\circ} \mathrm{C}$. Sections were thaw mounted on Super Frost Plus slides (Menzel, Braunschweig, Germany) and stored at $-70{ }^{\circ} \mathrm{C}$ until use. With few modifications, in situ hybridization experiments were performed using the protocol described in detail previously $(41,43)$. Briefly, sections were taken out from the $-70{ }^{\circ} \mathrm{C}$ freezer and immediately transferred into fixation solution $(4 \%$ paraformaldehyde in $0.1 \mathrm{M} \mathrm{NaHCO}_{3}, \mathrm{pH}$ 9.5) for 22 min at $4{ }^{\circ} \mathrm{C}$. Next, sections were washed in $1 \times$ PBS $\left(0.85 \% \mathrm{NaCl}, 1.4 \mathrm{mM} \mathrm{KH} \mathrm{PO}_{4}, 8 \mathrm{mM} \mathrm{Na}_{2} \mathrm{HPO}_{4}, \mathrm{pH}\right.$ 7.1) for $1 \mathrm{~min}$, incubated in $0.2 \mathrm{M} \mathrm{HCl}$ for $10 \mathrm{~min}$ and washed twice in 1xPBS for $2 \mathrm{~min}$ each. Then sections were incubated for $10 \mathrm{~min}$ in acetylation solution $(0.25 \%$ acetic anhydride freshly added in $0.1 \mathrm{M}$ triethanolamine) followed by 3 wash steps in $1 \times$ PBS (each wash step lasted $3 \mathrm{~min}$ ). Sections were incubated in pre-hybridization solution [5xSSC $(0.75$ $\mathrm{M} \mathrm{NaCl}, 0.075 \mathrm{M}$ sodium citrate, $\mathrm{pH} 7.0$ ) and $50 \%$ 
formamid] for $15 \mathrm{~min}$ at $4{ }^{\circ} \mathrm{C}$. For non-fluorescent in situ hybridization, each slide was subsequently covered with $100 \mu$ hybridization solution 1 [50\% formamide, $25 \% \mathrm{H}_{2} \mathrm{O}, 25 \%$ Microarray Hybridization Solution Version 2.0 (GE Healthcare, Freiburg, Germany)] containing the labeled antisense RNA probe. After placing a coverslip, slides were incubated in a humid box (50\% formamide) at $60{ }^{\circ} \mathrm{C}$ overnight. Visualization of digoxigenin-labeled probes in non-fluorescent in situ hybridization experiments was performed as described earlier (41) by means of an anti-digoxigenin alkaline phosphatase-conjugated antibody (Roche Diagnostics) diluted 1:750 and a substrate solution containing NBT (nitroblue tetrazolium) and BCIP (5-brom-4-chlor-3-indolyl phosphate). Tissue sections were analyzed with an Axioskop2 MOT (Carl Zeiss MicroImaging, Göttingen, Germany) equipped with an AxioCam MRc5 camera (Carl Zeiss) and AxioVision SE64 Rel. 4.9 software (Carl Zeiss).

For two-color fluorescent in situ hybridization (FISH), fixation, acetylation and hybridization were carried out as described above. Sections were hybridized with digoxigenin- and biotin-labeled probes simultaneously. However, for two-color FISH, $100 \mu$ hybridization solution $2(50 \%$ formamide, 2xSSC, $10 \%$ dextran sulphate, $0.2 \mathrm{mg} / \mathrm{ml}$ yeast $\mathrm{t}-\mathrm{RNA}$, $0.2 \mathrm{mg} / \mathrm{ml}$ herring sperm DNA) supplemented with labeled antisense RNA was placed per slide onto the tissue sections. Visualization of labeled probes was performed as described previously (50). In short, digoxigenin-labeled probes were visualized by the anti-digoxigenin alkaline phosphatase-conjugated antibody in combination with the HNPP fluorescent detection set (Roche Diagnostics). Incubation with the anti-digoxigenin alkaline phosphatase-conjugated antibody as well as incubation with the HNPP/Fast Red substrate were conducted overnight at $4{ }^{\circ} \mathrm{C}$. For visualization of biotin-labeled probes, the TSA fluorescein system kit (Perkin Elmer, Waltham, MA, USA) was used. Incubation of sections with biotin-binding streptavidin conjugated to horse radish peroxidase and incubation with fluoresceinconjugated tyramides were conducted overnight at 4 ${ }^{\circ} \mathrm{C}$. Sections were analyzed for fluorescent hybridization signals using a LSM 510 Meta laser scanning microscope (Carl Zeiss). Confocal image stacks were recorded from antennal segments. The micrographs shown represent selected optical planes.

\section{Results}

\section{Identification of sequences encoding candidate ORs in Schistocerca gregaria}

To identify sequences coding for SgreOR types, iterative tBLASTx searches in a Schistocerca gregaria antennal transcriptome database were performed using OR sequences from several other insect species. The putative OR-encoding sequences obtained from Schistocerca gregaria were further screened by BLASTx exploitations using the NCBI database. This approach led to the identification of 119 candidate SgreOR sequences of various lengths. These SgreORs were designated consecutively with Arabic numerals (SgreOR1 to SgreOR119). Naming of SgreOR sequences was conducted mainly in accordance with OR sequences of the related species Locusta migratoria for which 142 candidate ORs (termed LmigOrs) have been reported recently (48). Of the 119 identified candidate SgreOR sequences, 18 contained full length open reading frames (with a stop codon preceeding the presumptive start codon). Based on their length and their sequence, the open reading frames of further 6 sequences are putatively full length (they lack a stop codon preceeding the presumptive start codon). The remaining 95 SgreOR sequences presumably represent only partial sequences (more detailed information about the length of the SgreOR sequences and their GenBank accession numbers is given in supplementary table 2 and supplementary table 3 ). As a first step to analyze and characterize the SgreOR sequences, the deduced amino acid sequences of 117 SgreORs and 138 LmigOrs were used to generate a neighbor-joining tree (Fig. 1) (for these analyses, two SgreORs and four LmigOrs that represented only very short and partial OR sequences were excluded). Rooting of the tree was conducted with sequences from five different insect species that encode the odorant receptor co-receptor Orco, a non-canonical member of the OR family highly conserved among insects $(16,17)$. In the resulting phylogenetic tree, ORs from both species were arranged in several distinct groups of different sizes. Within the tree, for numerous SgreORs and LmigOrs, an orthologous sequence in the other species was found. The amino acid sequence identities for orthologous SgreORs/LmigOrs pairs were up to $\sim 90 \%$. However, for several SgreORs, no clear orthologue from Locusta migratoria was identified (e.g. SgreOR89, SgreOR101 and SgreOR111). In some cases, such SgreORs were part of small groups of paralogous sequences (for instance SgreOR94, SgreOR95 and SgreOR96). Vice versa, also for a number of LmigOrs, no orthologue from Schistocerca gregaria was observed (such as LmigOr78, LmigOr91 and LmigOr101) and some of these sequences belonged to small groups of paralogous LmigOrs (for example LmigOr20, LmigOr21, LmigOr22). 


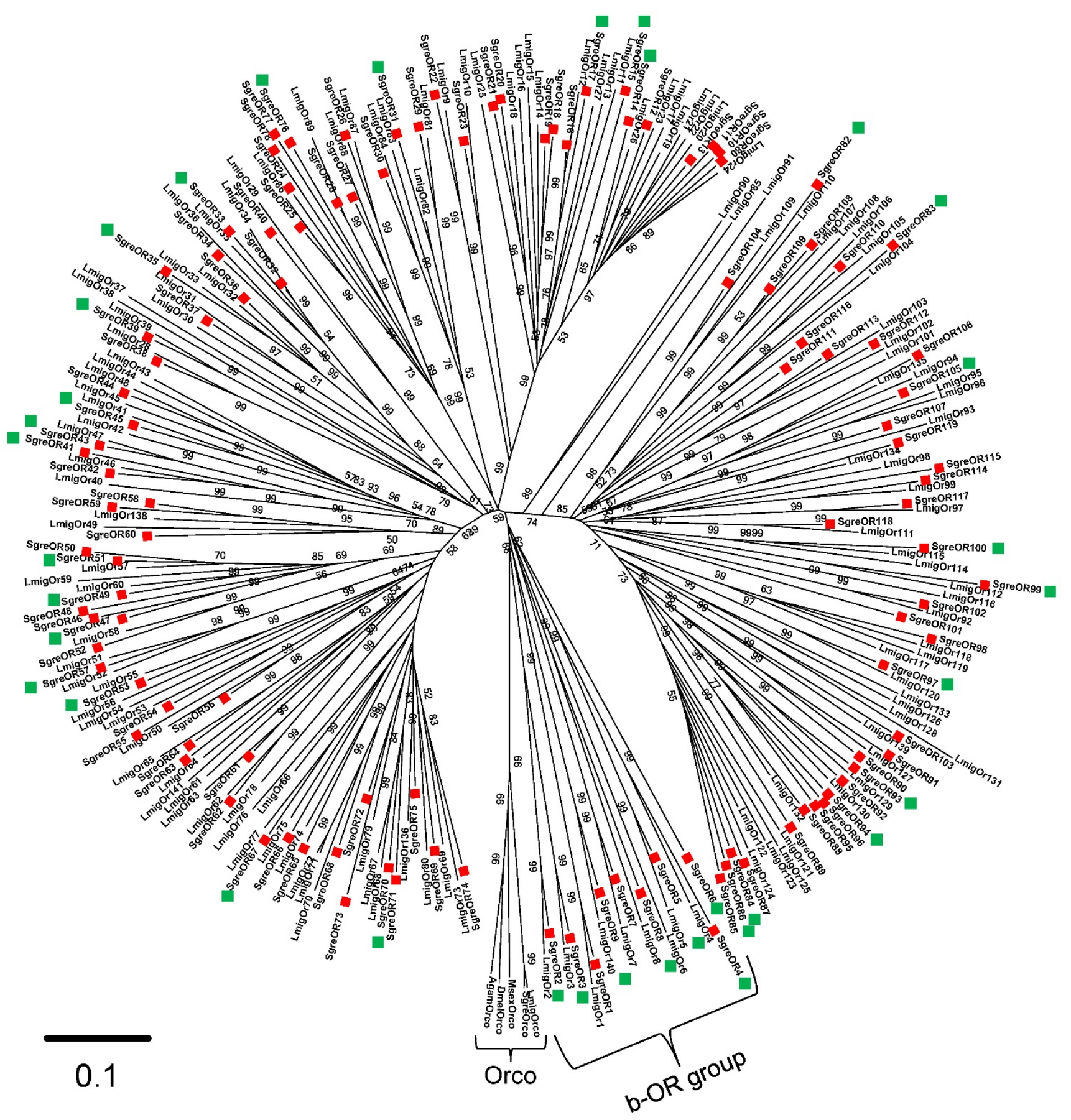

Fig. 1. Evolutionary relationships among SgreORs and LmigOrs. A neighbor-joining tree was calculated using the MEGA program. For rooting the tree, Orco-encoding sequences of different insect species were used. Calculations are based on a Clustal X alignment of the 117 SgreOR and 138 LmigOr amino acid sequences included in the tree. Branch lengths are proportional to the percentage of sequence differences. The scale bar indicates $10 \%$ difference. The numbers in the tree indicate bootstrap support values (in \%) based on 1000 replicates (only values above $50 \%$ are shown). OR-coding sequences from Schistocerca gregaria are denoted by red squares. SgreOR sequences used in two-color FISH experiments together with a SNMPI-specific probe are highlighted with green squares.

In the tree, one group of ORs including SgreOR1 to SgreOR9, LmigOr1 to LmigOr8 and LmigOr140 appeared to be unique due to some characteristic features. In particular, this group of ORs was more closely related to the Orco-encoding sequences at the root (basis) of the tree than other SgreORs, i.e. this group was situated at a phylogenetically more basal site than the other SgreORs (Fig. 1). Therefore, this group was designated as the basal OR (b-OR) group. It comprises 8 pairs of orthologous SgreORs/ LmigOrs. Within these pairs, the orthologues shared amino acid sequence identities between 47 and $87 \%$, indicating pronounced sequence conservation across species borders. The clear separation from other OR 
sequences in neighbor-joining trees and high sequence identity between species is reminescent of pheromone receptors from lepidopteran species, such as the tobacco budworm Heliothis virescens (3), the silkworm Bombyx mori (15), the tobacco hornworm Manduca sexta (51) or the cotton leafworm Spodoptera littoralis (52). However, no substantial sequence identities between b-ORs and known pheromone receptors from other insect species were found (data not shown).

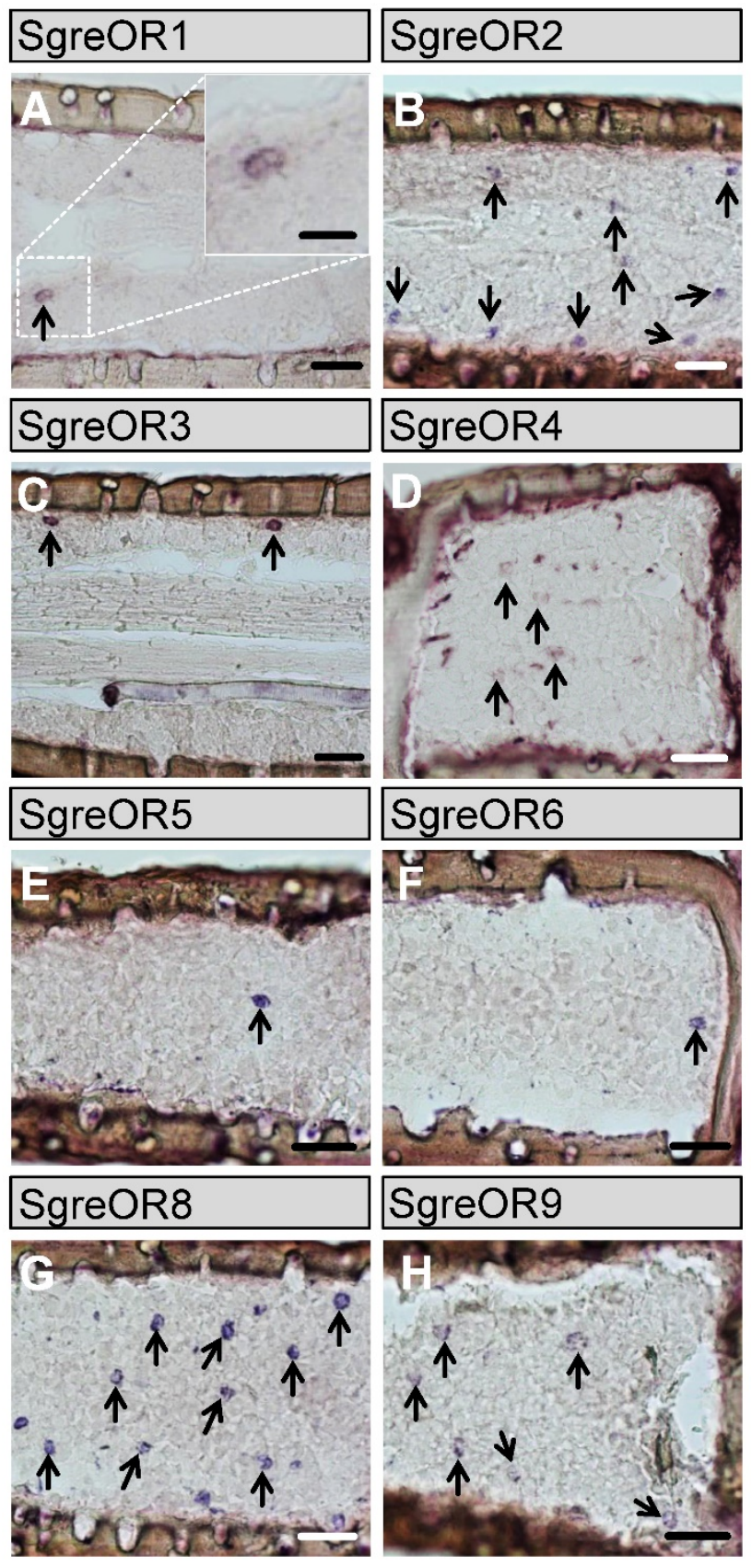

Fig. 2. Expression of the b-OR types in the antenna of Schistocerca gregaria. (A-H) In situ hybridizations were performed on longitudinal tissue sections of antennae with antisense RNA probes for the b-OR types indicated. Some of the stained cells are exemplarily denoted by arrows. Scale bars: $40 \mu \mathrm{m}$. Scale bar in the inset in A: $20 \mu \mathrm{m}$.

\section{Topographic expression of b-OR genes in the antennae of Schistocerca gregaria}

To analyze the expression of b-ORs in the antenna in more detail, PCR experiments with antennal cDNA from Schistocerca gregaria and specific primer pairs for all 9 members (SgreOR1 through SgreOR9) of the b-OR group of this species were conducted (data not shown). Cloning and sequencing of the resulting PCR products showed that they indeed encoded SgreOR1 to SgreOR9, further supporting the expression of these receptors in antennal tissue from Schistocerca gregaria.

For subsequent in situ hybridization experiments, RNA antisense probes were generated for the 9 members of the b-OR group. The results of these approaches revealed that distinct b-OR genes were expressed in varying numbers of cells in the antennae of Schistocerca gregaria (Fig. 2). No obvious differences were detected in the antennal expression patterns between males and females (data not shown). The receptor types SgreOR2 (Fig. 2B) and SgreOR8 (Fig. 2G) were expressed in a rather high number of cells per segment. Probes for the other b-ORs (SgreOR1, SgreOR3, SgreOR4, SgreOR5, SgreOR6 and SgreOR9) labeled only relatively small numbers of cells (Fig. 2A, Fig. 2C-F and Fig. 2H) suggesting that only few antennal cells express these receptors. We only observed very weak signals when in situ hybridization experiments were performed on antennal tissue sections with an antisense probe for SgreOR7 (data not shown).

\section{Co-expression of b-ORs with SNMP 1}

As an initial step to examine whether members of the b-OR group may represent candidate pheromone receptors, we used a strategy based on the observation that pheromone-responsive OSNs in insects express SNMP1 (20-25). In this regard, we have shown recently that SNMP1 is also expressed in a subpopulation of OSNs from Schistocerca gregaria (42). To approach the question whether members of the b-OR group may be co-expressed with SNMP1, two-color FISH experiments were performed with a biotin-labeled probe for SNMP1 and specific digoxigenin-labeled probes for distinct b-OR types from Schistocerca gregaria (SgreOR2, SgreOR3, SgreOR4, SgreOR5, SgreOR6, SgreOR8 and SgreOR9). In such experiments, SNMP1 was not co-expressed with SgreOR4 (supplementary Fig. 1A). By contrast, expression of SNMP1 was observed in cells positive for SgreOR2, SgreOR3, SgreOR5, SgreOR6, SgreOR8 (Fig. 3) and SgreOR9 (supplementary Fig. 1B). For unknown reason, it was not possible to visualize expression of SgreOR1 and SgreOR7 in two-color FISH experiments. Taken together, these findings 
demonstrate that most receptors of the b-OR group are co-expressed with SNMP1, suggesting that they

\section{SgreOR2/SNMP1}
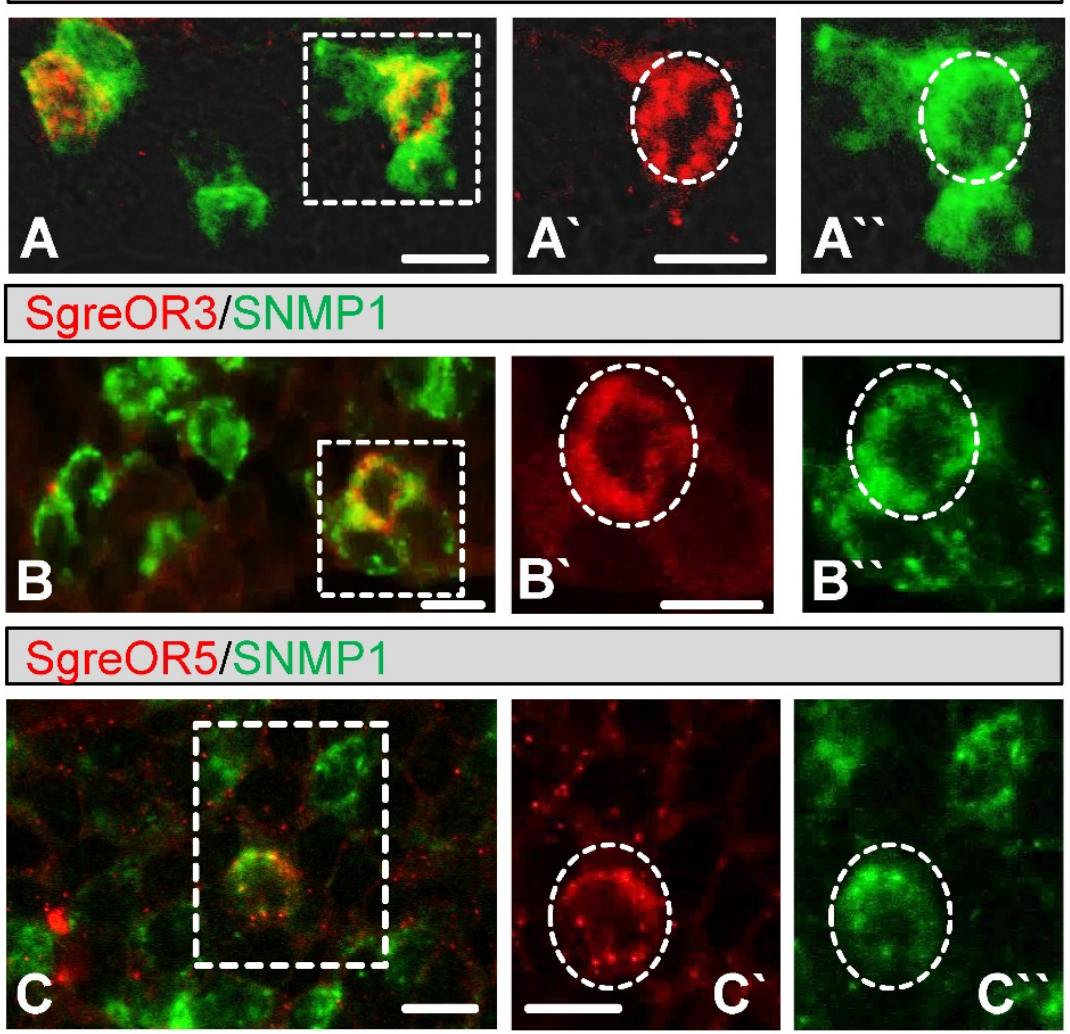

\section{SgreOR6/SNMP1}
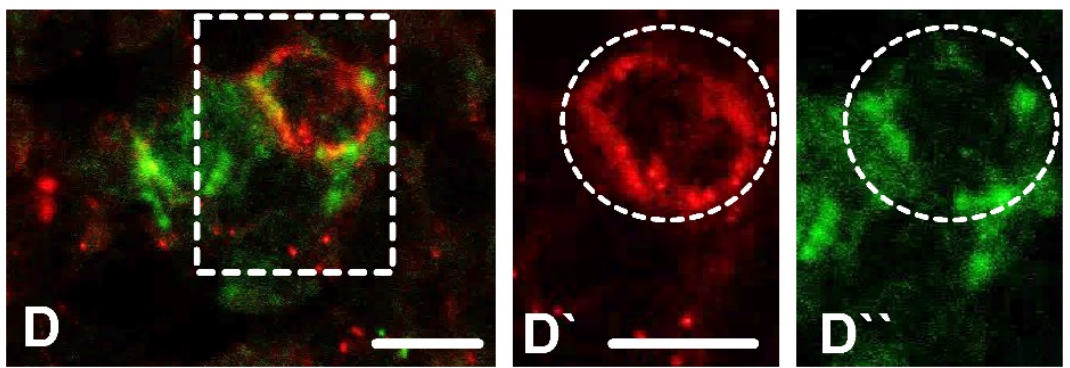

\section{SgreOR8/SNMP1}
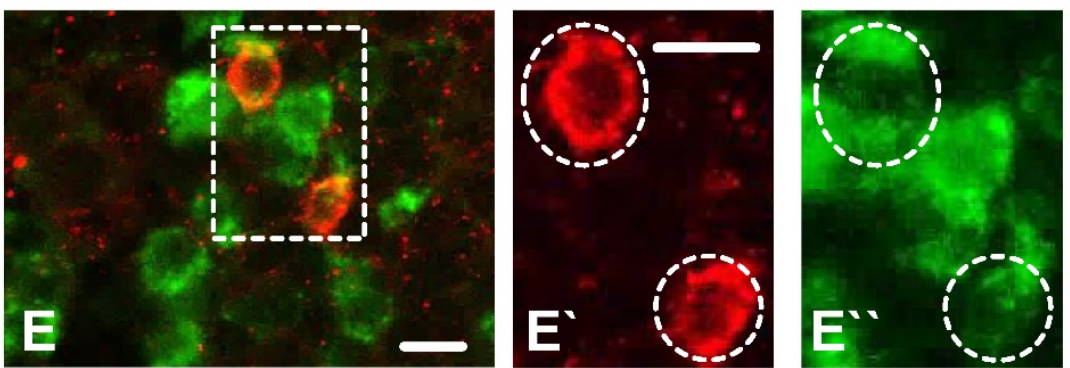

Fig. 3. Co-expression of different b-OR types with SNMPI. (A-E) Two-color FISH approaches were performed on sections through antennae with antisense RNA probes for SNMPI (A-E, green) and SgreOR2 (A, red), SgreOR3 (B, red), SgreOR5 (C, red), SgreOR6 (D, red) and SgreOR8 (E, red). The micrographs shown represent single optical planes taken from stacks of confocal images. Cells positive for SgreOR2, SgreOR3, SgreOR5, SgreOR6 or SgreOR8 co-express SNMP1. (A'IA“'-E'/E') Higher magnifications of the boxed areas in A, B, C, D and E are depicted in either the red or the green color channel. Cells co-expressing the relevant SgreOR types and SNMPI are marked with dashed circles. Scale bars: $10 \mu \mathrm{m}$ might function as pheromone receptors.

In two-color FISH approaches, several SNMP1-expressing neurons were frequently found to be located in close proximity to each other. The notion that all cells of such a small cluster of SNMP1-positive neurons may express the same b-OR type was not supported by our experiments. Instead, we found that only one cell in such a cluster expresses a given receptor of the b-OR group (Fig. 3).

\section{Sensilla-specific expression of b-OR group members}

SNMP1-expressing OSNs of Schistocerca gregaria, are found in sensilla trichodea and in sensilla basiconica (42). Consistently, in electrophysiological recordings from Schistocerca gregaria antennae, responses of both sensilla trichodea and sensilla basiconica to pheromonal substances have been observed (38). In a next step, we therefore set out to explore whether distinct receptors of the $b-O R$ group can be assigned to these types of sensilla. To address this issue, we performed two-color FISH experiments employing digoxigeninlabeled probes for distinct b-ORs (SgreOR2, SgreOR3, SgreOR5, SgreOR6, SgreOR8 and SgreOR9) and a biotin-labeled probe for Orco since OSNs beneath sensilla trichodea and sensilla basiconica express Orco $(41,42)$. It was found that cells expressing the receptor types SgreOR2 (Fig. 4A), SgreOR8 (Fig. 4B), SgreOR5, SgreOR6 and SgreOR9 (supplementary Fig. 2) were situated in the Orco-positive neuron clusters characteristic of sensilla basiconica. By contrast, cells expressing SgreOR3 were located directly beneath trichoid sensilla (Fig. 4C) and SgreOR3-positive neurons were only found within small groups of 2-3 Orco-expressing cells, an arrangement typical for sensilla trichodea (Fig. 4D). It is worthy of note that labeling with the Orco-specific probe was generally less intense in OSNs from trichoid sensilla as compared to neurons from sensilla basiconica (Fig. 4D). 


\section{SgreOR2/Orco}

\section{SgreOR8/Orco}
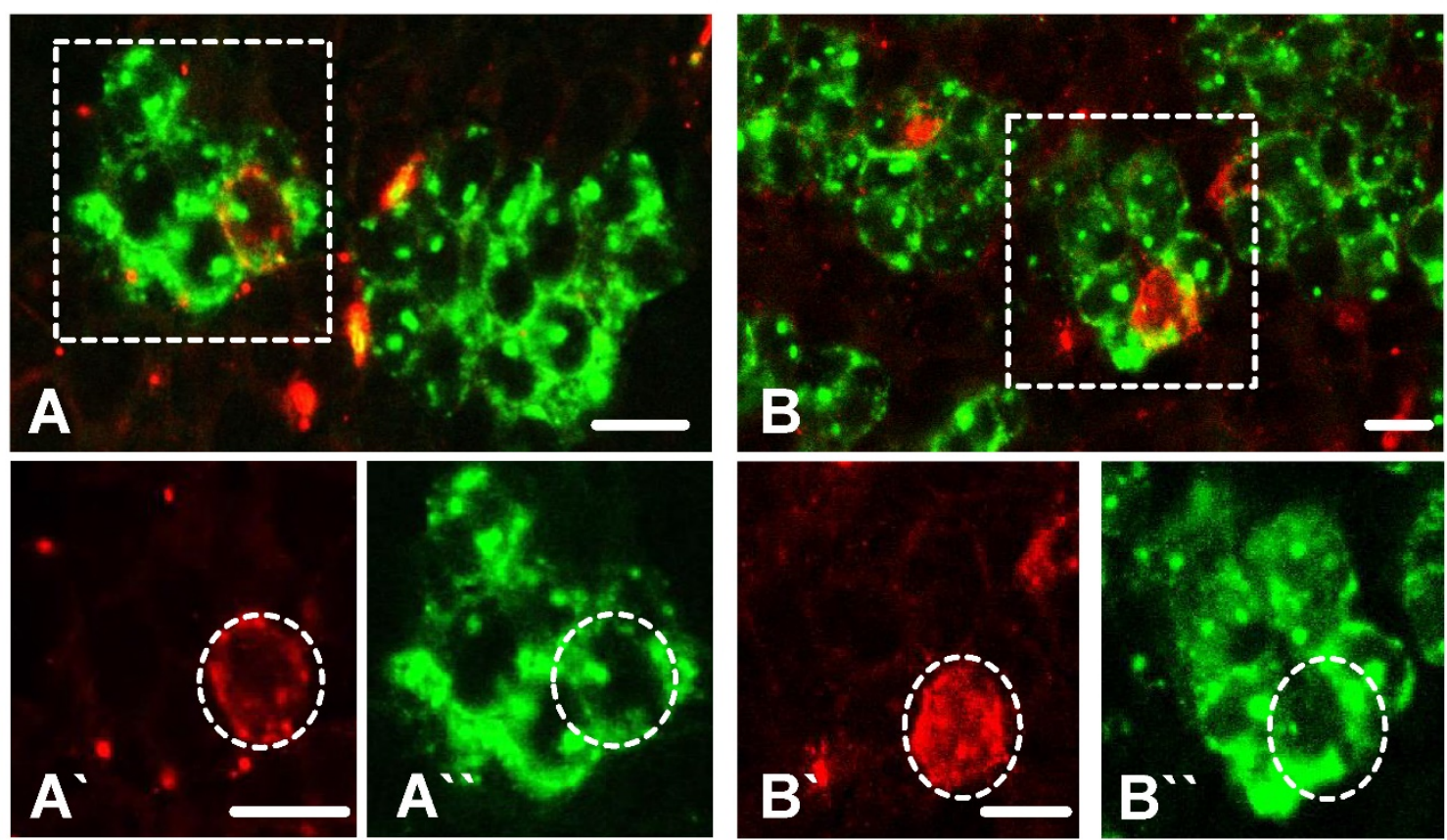

\section{SgreOR3}

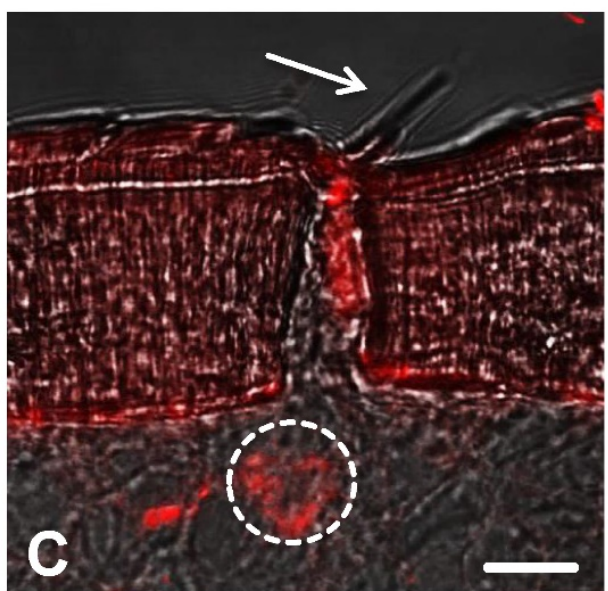

\section{SgreOR3/Orco}

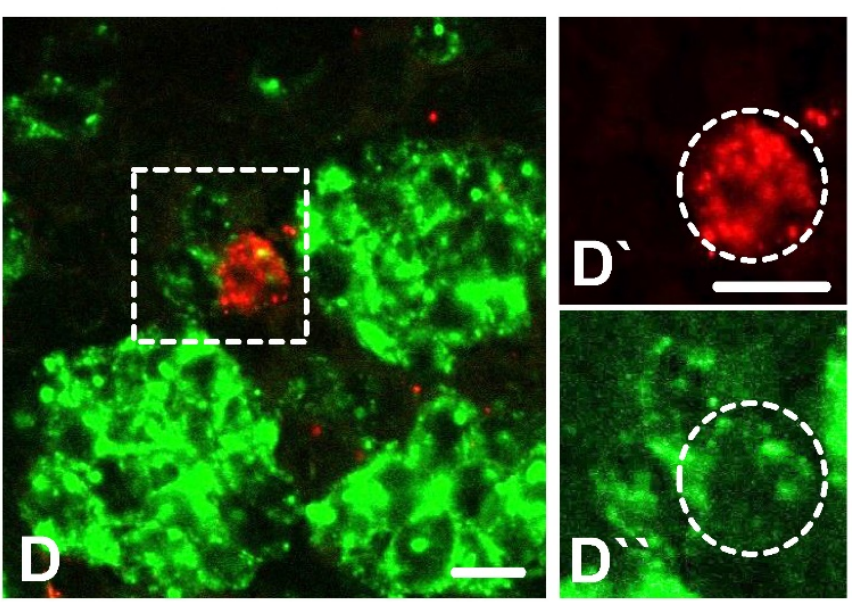

Fig. 4. Expression of distinct b-OR types in Orco-positive OSNs of sensilla basiconica or sensilla trichodea. (A-B) Two-color FISH experiments on sections through antennae were conducted with labeled antisense RNA probes for Orco (A-B, green) and SgreOR2 (A, red) or SgreOR8 (B, red). Higher magnifications of the regions indicated by dashed boxes are shown for the green and the red color channel in $A^{\prime}$ - $A^{\prime \prime}$ and $B^{\prime}$ - $B^{\prime \prime}$. Cells expressing SgreOR2 or SgreOR8 are located in the Orco-positive neuron clusters characteristic of sensilla basiconica. (C) FISH on antennal tissue employing an antisense RNA probe for SgreOR3 (red). A SgreOR3-positive labeled cell (dashed circle) is located directly below a sensillum trichodeum (arrow). (D) Two-color FISH with antisense probes for SgreOR3 (red) and Orco (green). As exemplarily depicted, SgreOR3-positive neurons were only found within small groups of Orco-expressing cells that are typical for sensilla trichodea. The area circumscribed by the dotted box in D is given in the green and the red color channel in D'-D ". Scale bars: $10 \mu \mathrm{m}$.

\section{Antennal expression and co-expression with SNMPI of SgreOR types outside the b-OR group}

To compare the expression pattern of the b-OR group members with SgreOR types not belonging to this group and to get a more detailed insight into the expression of SgreORs in the antennae of Schistocerca gregaria, we designed specific primer pairs for 28
SgreOR types (indicated in black font color in table 1) beyond the b-OR group and used them for PCR experiments with antennal cDNA from Schistocerca gregaria. Cloning and sequencing of the obtained PCR products demonstrated that they encoded the relevant receptors, further supporting expression of these SgreOR types in antennae of Schistocerca gregaria. Next, we prepared specific RNA probes for several of these SgreOR types and conducted in situ 
hybridizations. Similar to the results obtained for b-ORs (Fig. 2), these experiments revealed expression of distinct SgreOR types in different numbers of cells (Fig. 5). While a few of these SgreORs tested were expressed in a relatively high number of cells (as exemplarily shown for SgreOR17 and SgreOR35 in Fig. 5A-B), for most of them, only lower numbers of
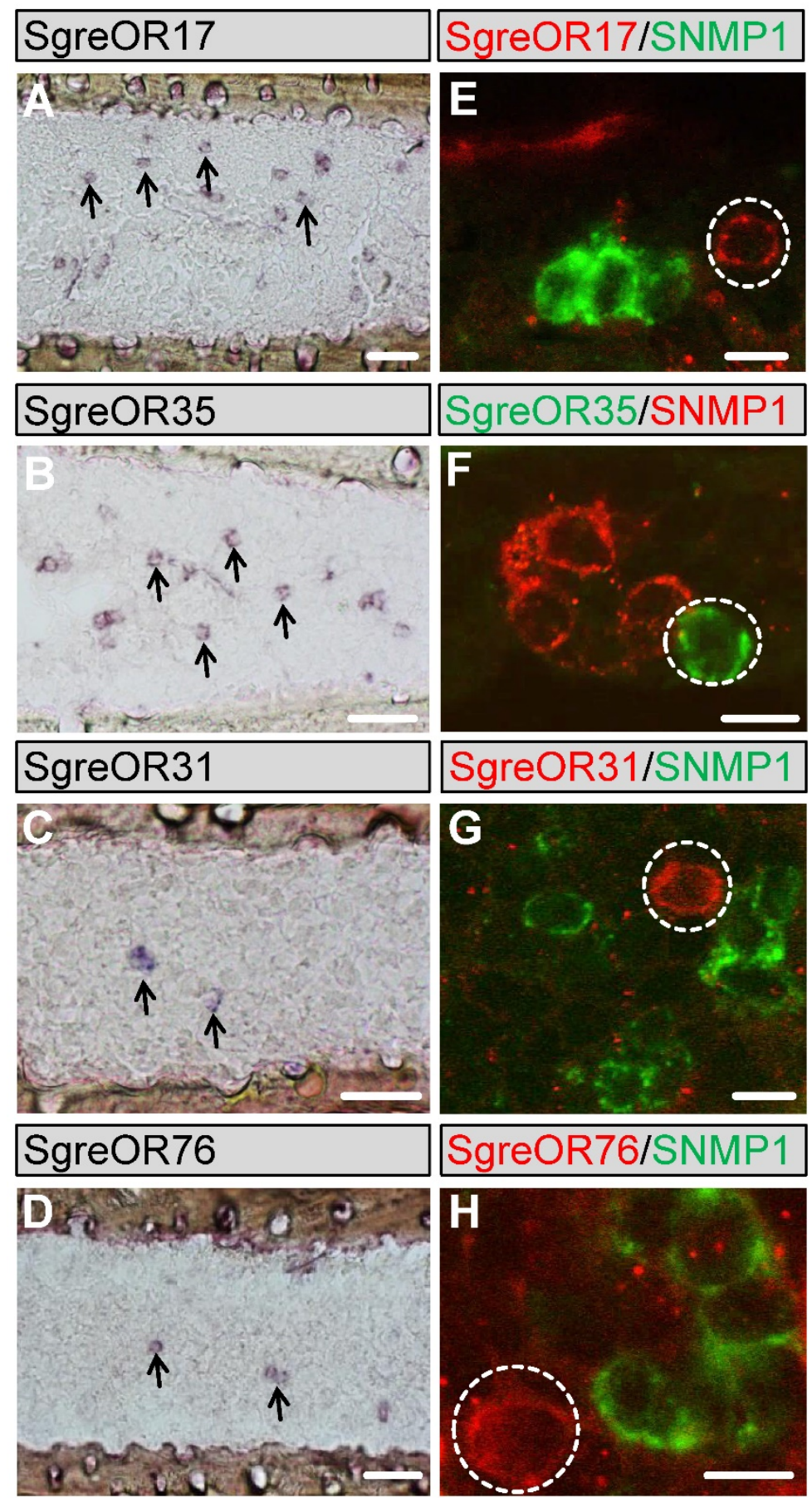

Fig. 5. Expression of SgreOR types outside the b-OR group in antennae of Schistocerca gregaria. (A-D) Non-fluorescent in situ hybridizations were conducted with probes for SgreOR17, SgreOR35, SgreOR31 and SgreOR76 on longitudinal antennal sections. Some of the stained cells are exemplarily denoted by arrows. (E-H) Two-color FISH experiments were performed with probes for the above-mentioned SgreOR types and SNMPI. Cells expressing these SgreORs (denoted by dashed circles) do not co-express SNMPI. Scale bars: A-C $40 \mu \mathrm{m}$; E-F $10 \mu \mathrm{m}$. stained cells were found per antennal section (as exemplarily depicted for SgreOR31 and SgreOR76 in Fig. 5C-D).

To test a potential co-expression of receptors outside the b-OR group with SNMP1, two-color FISH experiments were conducted with probes for SNMP1 and the 28 above-mentioned SgreOR types (these are also denoted by green squares in Fig. 1) that represent different branches of the neighbor-joining tree. The findings of these approaches are summarized in table 1 . For most of these SgreOR types, no co-expression with SNMP1 was detectable. This is exemplarily shown for SgreOR17, SgreOR31, SgreOR35, SgreOR76 (Fig. 5E-H) and SgreOR33, SgreOR45, SgreOR67, SgreOR82 (supplementary Fig. 3). However, for 5 of these SgreORs (SgreOR84, SgreOR86, SgreOR93, SgreOR94 and SgreOR97) co-expression with SNMP1 was observed (data not shown), indicating that there are SgreOR types outside the b-OR group that can be considered as candidate pheromone receptors. Yet, the percentage of SgreORs outside the b-OR group that are co-expressed with SNMP1 (5 out of 28 receptors tested; $\sim 22 \%$ ) was clearly lower than for the members of the b-OR group (6 out of 7 receptors tested; $\sim 86 \%$ ).

Table 1. Summary of the examined SgreOR types co-expressed or not co-expressed with SNMP1. According to the results of two-color FISH experiments, 6 of the 7 b-OR types (highlighted as italic in this table) tested $(\sim 86 \%)$ were found to be co-expressed with SNMPI (only SgreOR4 was not co-expressed with SNMPI). Regarding the SgreORs outside the b-OR group, 5 of 28 receptors examined $(\sim 22 \%)$ were co-expressed with SNMPI.

\begin{tabular}{lllll}
\hline $\begin{array}{l}\text { co-expressed with } \\
\text { SNMP1 }\end{array}$ & \multicolumn{3}{l}{ not co-expressed with SNMP1 } \\
\hline OR2 & OR3 & OR4 & OR14 & OR15 \\
OR5 & OR6 & OR17 & OR31 & OR33 \\
OR8 & OR9 & OR35 & OR39 & OR41 \\
OR84 & OR86 & OR43 & OR45 & OR47 \\
OR93 & OR94 & OR49 & OR51 & OR53 \\
OR97 & & OR57 & OR67 & OR70 \\
& & OR76 & OR82 & OR83 \\
& & OR99 & OR100 & OR105 \\
\hline
\end{tabular}

\section{Discussion}

In the present study, attempts have been made to identify candidate pheromone receptors of the desert locust Schistocerca gregaria. So far, very little is known about pheromone signaling in locusts. Therefore, 
we have used a novel approach based on the concept that pheromone-responsive OSNs in insects express SNMP1 $(20,21,24)$. Since its discovery $(46)$, SNMP1 has been considered as an accessory protein intimately involved in the recognition of pheromones $(47,53)$. This view was supported by recent findings underlining the importance of SNMP1 for the sensitivity and kinetics of a pheromone detection system $(25,54,55)$. These observations have led to the concept of a tunneling mechanism that transfers pheromones via SNMP1 to specific pheromone receptors implying an interplay between SNMP1 and receptors for pheromones (25). Consequently, ORs co-expressed with SNMP1 in antennal neurons may be considered as candidate pheromone receptors. In this study, we have identified 119 candidate OR genes from Schistocerca gregaria. Performing a phylogenetic comparison of the encoded OR proteins with ORs from Locusta migratoria, a relatively small group of receptors appeared to be unique due to its more basal position in the tree. Although the phylogenetic separation of these basal ORs (b-ORs) from other SgreORs is reminiscent of the results described for pheromone receptors in moth species $(15,51)$, no evident sequence identities between b-ORs and pheromone receptors from other insects were found. Overall, the missing sequence identities to moth and fly pheromone receptors do not argue against a potential function of b-ORs in pheromone reception and could be due to the large phylogenetic distance between the hemimetabolic Orthoptera (including locusts) on the one hand and the holometabolic Lepidoptera and Diptera on the other hand (33).

The results of two-color FISH experiments revealed that most of the analyzed b-ORs were co-expressed with SNMP1 and may therefore be considered as candidate pheromone receptors. Although the current data strongly support a role of SNMP1 in pheromone detection $(20,22,25,54,55)$, we are aware that verification of the SNMP1-coexpressed SgreOR types as pheromone receptors requires functional analyses. Thus, we cannot exclude the possibility that some of the SgreORs co-expressed with SNMP1 are used to detect non-pheromonal compounds. In this context, a member of the b-OR group from Locusta migratoria [named LmigOR3 (56); corresponding to LmigOr7 in the nomenclature used in the present study] has been recently found to be activated by food odorants (57). However, it remains unclear whether its orthologue from Schistocerca gregaria (SgreOR7) is co-expressed with SNMP1 since our two-color FISH experiments on antennal tissue using probes for SgreOR7 and SNMP1 were not successful.
Unlike pheromone receptors of moth and fly species (20-22,55), Schistocerca gregaria OSNs co-expressing SNMP1 and b-OR types were not primarily confined to trichoid sensilla. Instead, expression of most b-ORs was observed in OSNs beneath sensilla basiconica. This finding is however in accordance with previous electrophysiological recordings from Schistocerca gregaria antennae that demonstrated the responsiveness of both sensilla trichodea and sensilla basiconica to pheromone compounds (38).

In the desert locust, the remarkable change between the solitary and the gregarious phase seems to be controlled by signaling compounds (1). Thus, in Schistocerca gregaria, pheromones probably do not only play a central role in the reproduction process $(31,32)$ but also may be essential for the phase change. In line with the existence of various pheromonal compounds for eliciting different behaviors or priming diverse physiological effects in desert locusts, our two-color FISH experiments revealed further SgreOR types that alike the b-OR group members were co-expressed with SNMP1 and thus can also be considered as candidate pheromone receptors. While the b-ORs are more closely related to Orco than to other members of the SgreOR family, almost half of the putative pheromone receptors from Schistocerca gregaria identified in this study (SgreOR84, SgreOR86, SgreOR93, SgreOR94 and SgreOR97; table 1) belong to an OR group that is only distantly related to Orco (Fig. 1). It remains elusive why Schistocerca gregaria has two distinct groups of candidate pheromone receptors. Potentially, these two groups are dedicated to the detection of distinct types of pheromones.

In conclusion, uncovering candidate pheromone receptors of locusts could open the door for extended future studies including the screening for novel pheromonal compounds using heterologously expressed candidate pheromone receptors as well as the identification of receptors for previously verified pheromones $(1,31,36)$. Finally, in this context, the identification of SgreOR types mediating the detection of pheromones involved in reproduction and/or aggregation might provide important targets for inhibiting the formation of huge locust swarms that massively threaten agricultural crops in numerous countries.

\section{Abbreviations}

b-ORs: odorant receptors of the basal group; bp: base pairs; FISH: fluorescent in situ hybridization; LmigOrs: odorant receptors of Locusta migratoria; ORs: odorant receptors; OSNs: olfactory sensory neurons; SgreORs: odorant receptors of Schistocerca gregaria; SNMP1: sensory neuron membrane protein 1 . 


\section{Supplementary Material}

Supplementary figures and tables.

http://www.ijbs.com/v13p0911s1.pdf

\section{Acknowledgements}

The authors would like to thank Heidrun Froß and Kathrin Dietze for excellent technical assistance.

\section{Funding}

Xingcong Jiang was supported by a grant financed by the China Scholarship Council (CSC).

\section{Data deposition}

The SgreOR sequences reported in this study have been deposited in GenBank [accession numbers KY964918 (SgreOR1) through KY965036 (SgreOR119)].

\section{Competing Interests}

The authors have declared that no competing interest exists.

\section{References}

1. Hassanali A, Njagi PGN, Bashir MO. Chemical ecology of locusts and related acridids. Annu Rev Entomol. 2005; 50: 223-45.

2. Hansson BS, Stensmyr MC. Evolution of insect olfaction. Neuron. 2011; 72: 698-711.

3. Krieger J, Grosse-Wilde E, Gohl T, Dewer YME, Raming K, Breer H. Genes encoding candidate pheromone receptors in a moth (Heliothis virescens). Proc Natl Acad Sci U S A. 2004;101: 11845-50

4. Nakagawa T, Sakurai T, Nishioka T, Touhara K. Insect sex-pheromone signals mediated by specific combinations of olfactory receptors. Science. 2005; 307: 1638-42.

5. Grosse-Wilde E, Gohl T, Bouche E, Breer H, Krieger J. Candidate pheromone receptors provide the basis for the response of distinct antennal neurons to pheromonal compounds. Eur J Neurosci. 2007; 25: 2364-73.

6. Kurtovic A, Widmer A, Dickson BJ. A single class of olfactory neurons mediates behavioural responses to a Drosophila sex pheromone. Nature. 2007; 446: 542-6.

7. Almaas TJ, Mustaparta H. Heliothis virescens: Response characteristics of receptor neurons in Sensilla Trichodea type 1 and type 2. J Chem Ecol. 1991; 17: 953-72.

8. Baker TC, Ochieng SA, Cosse AA, Lee SG, Todd JL, Quero C, Vickers NJ. A comparison of responses from olfactory receptor neurons of Heliothis subflexa and Heliothis virescens to components of their sex pheromone. J Comp Physiol A. $2004 ; 190: 155-65$.

9. Keil T, Steinbrecht RA. Mechanosensitive and olfactory sensilla of insects. In: King R, Akai H, eds. Insect Ultrastructure, Volume 2, New York: Springer US; 1984: 477-516.

10. Steinbrecht RA, Gnatzy W. Pheromone receptors in Bombyx mori and Antheraea pernyi. I. Reconstruction of the cellular organization of the sensilla trichodea. Cell Tissue Res. 1984; 235: 25-34.

11. Keil A. Fine structure of the pheromone-sensitive sensilla on the antenna of the hawkmoth, Manduca sexta. Tissue Cell. 1989; 21: 139-51.

12. Clyne P, Grant A, O'Connell R, Carlson JR. Odorant response of individual sensilla on the Drosophila antenna. Invert Neurosci. 1997; 3: 127-35.

13. Couto A, Alenius M, Dickson BJ. Molecular, Anatomical, and Functional Organization of the Drosophila Olfactory System. Curr Biol. 2005; 15: 1535-47.

14. Sakurai T, Nakagawa T, Mitsuno H, Mori H, Endo $Y$, Tanoue $S$, Yasukochi $Y$, Touhara K, Nishioka T. Identification and functional characterization of a sex pheromone receptor in the silkmoth Bombyx mori. Proc Natl Acad Sci U S A. 2004; 101: 16653-8.

15. Krieger J, Grosse-Wilde E, Gohl T, Breer H. Candidate pheromone receptors of the silkmoth Bombyx mori. Eur J Neurosci. 2005; 21: 2167-76.

16. Krieger J, Klink O, Mohl C, Raming K, Breer H. A candidate olfactory receptor subtype highly conserved across different insect orders. J Comp Physiol A. 2003; 189: 519-26.

17. Larsson MC, Domingos AI, Jones WD, Chiappe ME, Amrein H, Vosshall LB. Or83b encodes a broadly expressed odorant receptor essential for Drosophila olfaction. Neuron. 2004; 43: 703-14.
18. Sato K, Pellegrino M, Nakagawa T, Nakagawa T, Vosshall LB, Touhara K. Insect olfactory receptors are heteromeric ligand-gated ion channels. Nature. 2008; 452: 1002-6.

19. Wicher D, Schäfer R, Bauernfeind R, Stensmyr MC, Heller R, Heinemann SH, Hansson BS. Drosophila odorant receptors are both ligand-gated and cyclic-nucleotide-activated cation channels. Nature. 2008; 452: 1007-11.

20. Benton R, Vannice KS, Vosshall LB. An essential role for a CD36-related receptor in pheromone detection in Drosophila. Nature. 2007; 450: 289-93.

21. Forstner M, Gohl T, Gondesen I, Raming K, Breer H, Krieger J. Differential expression of SNMP-1 and SNMP-2 proteins in pheromone-sensitive hairs of moths. Chem Senses. 2008; 33: 291-9.

22. Jin $\mathrm{X}, \mathrm{Ha}$ TS, Smith DP. SNMP is a signaling component required for pheromone sensitivity in Drosophila. Proc Natl Acad Sci U S A. 2008;105: 10996-1001.

23. Liu S, Zhang YR, Zhou WW, Liang QM, Yuan X, Cheng J, Zhu ZR, Gong ZJ. Identification and characterization of two sensory neuron membrane proteins from Cnaphalocrocis medinalis (Lepidoptera: Pyralidae). Arch Insect Biochem Physiol. 2013; 82: 29-42.

24. Zhang J, Liu Y, Walker WB, Dong S-L, Wang G-R. Identification and localization of two sensory neuron membrane proteins from Spodoptera litura (Lepidoptera: Noctuidae). Insect Sci. 2015; 22: 399-408.

25. Gomez-Diaz C, Bargeton B, Abuin L, Bukar N, Reina JH, Bartoi T, Graf M, Ong $\mathrm{H}$, Ulbrich MH, Masson JF, Benton R. A CD36 ectodomain mediates insect pheromone detection via a putative tunnelling mechanism. Nat Commun. 2016; 7: 11866.

26. Fuzeau-Braesch S, Genin E, Jullien R, Knowles E, Papin C. Composition and role of volatile substances in atmosphere surrounding two gregarious locusts, Locusta migratoria and Schistocerca gregaria. J Chem Ecol. 1988; 14: 1023-33.

27. Byers JA. Pheromones and chemical ecology of locusts. Biol Rev. 1991; 66: 347-78.

28. Obeng-Ofori D, Torto B, Njagi PGN, Hassanali A, Amiani H. Fecal volatiles as part of the aggregation pheromone complex of the desert locust, Schistocerca gregaria (Forskal) (Orthoptera: Acrididae). J Chem Ecol. 1994; 20: 2077-87.

29. Torto B, Obeng-Ofori D, Njagi PGN, Hassanali A, Amiani H. Aggregation pheromone system of adult gregarious desert locust Schistocerca gregaria (forskal). J Chem Ecol. 1994; 20: 1749-62.

30. Pener MP, Yerushalmi Y. The physiology of locust phase polymorphism: an update. J Insect Physiol. 1998; 44: 365-77.

31. Seidelmann K, Ferenz H-J. Courtship inhibition pheromone in desert locusts, Schistocerca gregaria. J Insect Physiol. 2002; 48: 991-6.

32. Stahr C, Svatos A, Seidelmann K. Chemical identification, emission pattern and function of male-specific pheromones released by a rarely swarming locust, Schistocerca americana. J Chem Ecol. 2013; 39: 15-27.

33. Misof B, Liu S, Meusemann K, Peters RS, Donath A, Mayer C, et al. Phylogenomics resolves the timing and pattern of insect evolution. Science. 2014; 346: 763-7.

34. Simpson SJ, Sword GA. Locusts. Curr Biol. 2008; 18: R364-6.

35. Heifetz Y, Voet H, Applebaum S. Factors affecting behavioral phase transition in the desert locust Schistocerca gregaria (Forskal) (Orthoptera: Acrididae). J Chem Ecol. 1996; 22: 1717-34.

36. Seidelmann K, Weinert H, Ferenz H-J. Wings and legs are production sites for the desert locust courtship-inhibition pheromone, phenylacetonitrile. J Insect Physiol. 2003; 49: 1125-33.

37. Hansson BS, Ochieng SA, Grosmaitre X, Anton S, Njagi PGN. Physiological responses and central nervous projections of antennal olfactory receptor neurons in the adult desert locust, Schistocerca gregaria (Orthoptera: Acrididae). J Comp Physiol A. 1996; 179: 157-67.

38. Ochieng SA, Hansson BS. Responses of olfactory receptor neurones to behaviourally important odours in gregarious and solitarious desert locust, Schistocerca gregaria. Physiol Entomol. 1999; 24:28-36.

39. Cui X, Wu C, Zhang L. Electrophysiological response patterns of 16 olfactory neurons from the trichoid sensilla to odorant from fecal volatiles in the locust, Locusta migratoria manilensis. Arch Insect Biochem Physiol. 2011; 77: 45-57.

40. Ochieng SA, Hallberg E, Hansson BS. Fine structure and distribution of antennal sensilla of the desert locust, Schistocerca gregaria (Orthoptera: Acrididae). Cell Tissue Res. 1998; 291: 525-36.

41. Yang Y, Krieger J, Zhang L, Breer H. The olfactory co-receptor Orco from the migratory locust (Locusta migratoria) and the desert locust (Schistocerca gregaria): identification and expression pattern. Int J Biol Sci. 2012; 8: 159-70.

42. Jiang X, Pregitzer P, Grosse-Wilde E, Breer H, Krieger J. Identification and Characterization of Two "Sensory Neuron Membrane Proteins" (SNMPs) of the Desert Locust, Schistocerca gregaria (Orthoptera: Acrididae). J Insect Sci. 2016; 16: 33

43. Guo M, Krieger J, Große-Wilde E, Mißbach C, Zhang L, Breer H. Variant ionotropic receptors are expressed in olfactory sensory neurons of coeloconic sensilla on the antenna of the desert locust (Schistocerca gregaria). Int J Biol Sci. 2014; 10: 1-14

44. Benton R, Vannice KS, Gomez-Diaz C, Vosshall LB. Variant ionotropic glutamate receptors as chemosensory receptors in Drosophila. Cell. 2009; 136: 149-62.

45. Rytz R, Croset V, Benton R. Ionotropic Receptors (IRs): Chemosensory ionotropic glutamate receptors in Drosophila and beyond. Insect Biochem Mol Biol. 2013; 43: 888-97. 
46. Rogers ME, Sun M, Lerner MR, Vogt RG. Snmp-1, a novel membrane protein of olfactory neurons of the silk moth Antheraea polyphemus with homology to the CD36 family of membrane proteins. J Biol Chem. 1997; 272: 14792-9.

47. Rogers ME, Steinbrecht RA, Vogt RG. Expression of SNMP-1 in olfactory neurons and sensilla of male and female antennae of the silkmoth Antheraea polyphemus. Cell Tissue Res. 2001; 303: 433-46.

48. Wang Z, Yang P, Chen D, Jiang F, Li Y, Wang X, Kang L. Identification and functional analysis of olfactory receptor family reveal unusual characteristics of the olfactory system in the migratory locust. Cell Mol Life Sci. 2015; 72: 4429-43.

49. Tamura K, Stecher G, Peterson D, Filipski A, Kumar S. MEGA6: Molecular Evolutionary Genetics Analysis Version 6.0. Mol Biol Evol. 2013; 30: 2725-9.

50. Krieger J, Raming K, Dewer YME, Bette S, Conzelmann S, Breer H. A divergent gene family encoding candidate olfactory receptors of the moth Heliothis virescens. Eur J Neurosci. 2002; 16: 619-28.

51. Grosse-Wilde E, Kuebler LS, Bucks S, Vogel H, Wicher D, Hansson BS. Antennal transcriptome of Manduca sexta. Proc Natl Acad Sci U S A. 2011; 108: 7449-54.

52. Legeai F, Malpel S, Montagne N, Monsempes C, Cousserans F, Merlin C, François MC, Maibeche-Coisne M, Gavory F, Poulain J, Jacquin-Joly E. An Expressed Sequence Tag collection from the male antennae of the Noctuid moth Spodoptera littoralis: a resource for olfactory and pheromone detection research. BMC Genomics. 2011; 12: 86.

53. Vogt RG, Miller NE, Litvack R, Fandino RA, Sparks J, Staples J, Friedman R, Dickens JC. The insect SNMP gene family. Insect Biochem Mol Biol. 2009; 39: 448-56.

54. Li Z, Ni JD, Huang J, Montell C. Requirement for Drosophila SNMP1 for rapid activation and termination of pheromone-induced activity. PLoS Genet. 2014; 10: e1004600.

55. Pregitzer P, Greschista M, Breer H, Krieger J. The sensory neurone membrane protein SNMP1 contributes to the sensitivity of a pheromone detection system. Insect Mol Biol. 2014; 23: 733-42.

56. Xu H, Guo M, Yang Y, You Y, Zhang L. Differential expression of two novel odorant receptors in the locust (Locusta migratoria). BMC Neurosci. 2013; 14: 50.

57. You Y, Smith DP, Lv M, Zhang L. A broadly tuned odorant receptor in neurons of trichoid sensilla in locust, Locusta migratoria. Insect Biochem Mol Biol. 2016; pii: S0965-1748(16)30155-2. 